\title{
Effect of AQP4-RNAi in treating traumatic brain edema: Multi-modal MRI and histopathological changes of early stage edema in a rat model
}

\author{
YING GUAN ${ }^{1}$, LIFENG LI ${ }^{2}$, JIANQIANG CHEN ${ }^{3}$ and HONG LU ${ }^{4}$ \\ ${ }^{1}$ Department of Ultrasonography, The First Affiliated Hospital of Hainan Medical University, Haikou, Hainan 570102; \\ ${ }^{2}$ Department of Radiology, Changsha Central Hospital, Changsha, Hunan 410004; ${ }^{3}$ Department of Radiology, \\ Haikou People's Hospital, Haikou, Hainan 570208; ${ }^{4}$ Department of Radiology, \\ The Seventh People's Hospital of Chongqing, Chongqing 400054, P.R. China
}

Received September 13, 2018; Accepted August 30, 2019

DOI: $10.3892 /$ etm.2020.8456

\begin{abstract}
Traumatic brain injury (TBI) is one of the leading causes of mortality and permanent disabilities worldwide. Brain edema following TBI remains to be the predominant cause of mortality and disability in patients worldwide. Previous studies have reported that brain edema is closely associated with aquaporin-4 (AQP4) expression. AQP4 is a water channel protein and mediates water homeostasis in a variety of brain disorders. In the current study, a rat TBI model was established, and the features of brain edema following TBI were assessed using multimodal MRI. The results of the multimodal MRI were useful, reliable and were used to evaluate the extent and the type of brain edema following TBI. Brain edema was also successfully alleviated using an intracerebral injection of AQP4 small interfering (si)RNA. The expression of AQP4 and its role in brain edema were also examined in the present study. The AQP4 siRNA was demonstrated to downregulate AQP4 expression following TBI and reduced brain edema at the early stages of TBI (6 and $12 \mathrm{~h}$ ). The current study revealed the MRI features of brain edema and the changes in AQP4 expression exhibited following TBI, and the results provide important information that can be used to improve the early diagnosis and treatment of brain edema.
\end{abstract}

\section{Introduction}

The occurrence of traumatic brain injury (TBI) is increasing and has recently become one of the leading causes of mortality and permanent disability worldwide (1). In America, TBI contributes to $30 \%$ of all deaths associated with head trauma

Correspondence to: Dr Hong Lu, Department of Radiology, The Seventh People's Hospital of Chongqing, 1 One Village, Chongqing 400054, P.R. China

E-mail: hnguanying@163.com

Key words: aquaporin 4, multi-modal MRI, traumatic brain edema and 3.2 million disabilities each year (2). The disturbance of water homeostasis in the brain is an important pathological change in TBI, where brain edema following TBI remains to be the predominant cause of mortality and disability among patients admitted to the neurology department $(3,4)$. TBI can directly cause brain damage, and the resulting brain edema increases intracranial pressure and can lead to brain ischemia and mortality (5).

In the current study, a non-invasive diffusion-weighted MRI (DWI) was used to monitor brain edema following TBI. The use of DWI allows the identification of the types and characteristics of edema that can develop after TBI. Although numerous studies have investigated brain edema using DWI, the underlying mechanisms and specific time course of this accumulation remain controversial (6-8). Edema can be identified using DWI at the early stage of TBI, while the apparent diffusion coefficient (ADC) sequence accurately indicates the extent and range of edema (7). Multimodal MRI including DWI, T2 weighted image (T2WI), ADC and susceptibility weighted image (SWI) sequences may provide more information and provide a better understanding of brain edema (7).

Aquaporin-4 (AQP4) is a member of the aquaporin family, which acts as a water channel protein and mediates water homeostasis. Among the aquaporin family, AQP4 is the most important type in the central nervous system (9). Previous studies have indicated that AQP4 serves a key role in the formation and resolution of brain edema (10-13). The knockdown of AQP4 has also been indicated to be effective in improving brain disorders that are caused by edema (1). However, the early stage $(<12 \mathrm{~h})$ of brain edema and multi-modal MRI findings following AQP4 RNAi treatment are still unclear.

In the current study, the multi-modal MRI and histopathological changes were investigated at the early stage of traumatic brain edema in an in vivo model. The present study aimed to provide an improved understanding of the features of brain edema after TBI. With an improved understanding of brain edema after TBI, AQP4 expression was decreased in the brain using small interfering (si)RNA, and the effectiveness of AQP4 as a potential therapeutic target for brain edema was assessed. 


\section{Materials and methods}

Animals and siRNA synthesis. The current study was approved by the Ethics Committee for the First Affiliated Hospital of Hainan Medical University (approval no. 2016054; Hainan, China). All experimental procedures were conducted according to the Guidelines for Animal Experimentation of the First Affiliated Hospital of Hainan Medical University (Hainan, China). A total of 120 male Wistar rats (age, 9-12 weeks; weight, 250-300 g), were purchased from the Experimental Animal Center of the First Affiliated Hospital of Hainan Medical University (Hainan, China). Rats were housed under controlled conditions with a $12 \mathrm{~h}$ light/dark cycle, with $21 \pm 2^{\circ} \mathrm{C}$ and $60-70 \%$ humidity, and allowed free access to a standard dry rat diet and tap water ad libitum.

The AQP4-RNAi was designed and synthetized by GeneChem, Inc. The siRNA sequence was as follows: Sense, 5'-GCAGUUAUCAUGGGA AACUTT-3' and antisense, 5'-AGUUUCCCAUGAUAACUGCTT-3'. The control RNAi sequence was sense, 5'-UUCUCCGAACGUGUCACGU-3' and antisense, 5'-ACGUGACACGUUCGGAGAA-3'.

Groups and animal experiments. A total of 120 Wistar rats were divided into four groups (30 rats each) as follows: The control group (control), trauma group (trauma), placebo treatment group (placebo) and the AQP4-RNAi treatment group (RNAi).

The animal brain trauma model was established using a methodology previously described by McIntosh et al (12), using a PinPoint ${ }^{\mathrm{TM}}$ Precision Cortical Impactor (Hatteras Instruments, Inc.). Rats were anesthetized using isoflurane ( $4 \%$ for induction and $2 \%$ for maintenance). A $1.5 \mathrm{~mm}$ drill bit (rotational speed, 4,000 r/min) equipped with a table electric dental engine (cat. no. 307-2B; Shenzhen Ward Life Science and Technology Co., Ltd.) was used to bore a hole into the skull between the anterior and posterior fontanel. A $5 \mathrm{~mm}$-diameter round window was created using a mosquito clamp (cat. no. C5971A; Shenzhen Ward Life Science and Technology Co.,Ltd.) In the trauma, placebo and RNAi groups, the bony window was impinged using the impactor to establish moderate right brain trauma. The parameters were as follows: Impinging velocity, $2.5 \mathrm{~m} / \mathrm{sec}$; impinging depth, $4 \mathrm{~mm}$; duration, $0.85 \mathrm{sec}$; diameter of impinging bit, $4 \mathrm{~mm}$. In the control group, an identical opening in the skull was created but no impact was made. Rats received an intracerebroventricular injection 10 min after injury in the placebo and RNAi groups. A total of $10 \mu \mathrm{l}$ AQP4-RNAi (RNAi group) or control RNAi (placebo group) was injected into the lateral ventricle using a 30 -gauge needle on a Hamilton syringe at a final concentration of $20 \mathrm{nM}$. The Entranster ${ }^{\mathrm{TM}}-$ in vivo was used as the carrier of RNAi (Engreen Biosystem Co, Ltd.). MRI images of the rat brain were captured at 1,6 and $12 \mathrm{~h}$ after injury, following which the rats were immediately sacrificed after MRI imaging to obtain brain tissue samples. The rats were euthanized using $\mathrm{CO}_{2}$ at an air displacement rate of $20 \%$ per min.

Western blot analysis. Total protein was extracted using RIPA lysis buffer (Shanghai BestBio Biotechnology Co., Ltd.). Protein levels in tissue homogenates were quantified using a BCA Protein assay kit (Thermo Fisher Scientific, Inc.), and $10 \mu \mathrm{g}$ protein (each sample) was separated by SDS-PAGE (10\% gel). Proteins were then transferred onto $0.22-\mu \mathrm{m}$ PVDF membranes and blocked with $5 \%$ fat-free milk at room temperature for $2 \mathrm{~h}$. Membranes were subsequently incubated at $4^{\circ} \mathrm{C}$ overnight with primary antibodies against AQP4 (1:1,000; cat. no. ab46182; Abcam) and $\beta$-actin (1:1,000; cat. no. ab8227; Abcam). Membranes were then washed in Tris-buffered saline containing $0.05 \%$ Tween 20 (TBS-T) and incubated with horseradish peroxidase-conjugated anti-rabbit IgG antibodies (1:5,000; cat. no. 7076; CST Biological Reagents Co., Ltd.) for $1 \mathrm{~h}$ at room temperature. Membranes were then incubated with enhanced chemiluminescence reagent (ECL Western blotting kit; GE Healthcare Life Sciences). The positive bands were detected using a Bio-Rad Molecular Imager system (Bio-Rad Laboratories, Inc.). Densitometric analysis was performed using Quantity One 1-D software (version 4.6.9; Bio-Rad Laboratories, Inc.), and the target bands were normalized to $\beta$-actin.

MRI and image analysis. Rats were anesthetized, and all MRI measurements were collected in a 7.0T dedicated experimental animal scanner (Bruker BioSpin; Bruker Corporation) at 1,6 and $12 \mathrm{~h}$ following injury. The scan parameters were as follows: Localizer: TR $100.0 \mathrm{~ms}$, TE: $3.0 \mathrm{~ms}$, matrix: 256x256, depth of stratum $1.0 \mathrm{~mm}$, interlamellar spacing $0.00 \mathrm{~mm}$, FOV: $4.0 \times 4.0 \mathrm{~cm}$; T2WI: TR, $4000.0 \mathrm{~ms}$; TE, $35 \mathrm{~ms}$; matrix, 256x 256; depth of stratum, $1.0 \mathrm{~mm}$; interlamellar spacing, $0.00 \mathrm{~mm}$; FOV, 3.5x3.5 cm; SWI: TR, $700.0 \mathrm{~ms}$; TE, $12 \mathrm{~ms}$, matrix, $384 \times 384$; depth of stratum, $1.0 \mathrm{~mm}$; interlamellar spacing, $0.00 \mathrm{~mm}$; FOV, $3.5 \times 3.5 \mathrm{~cm}$; DWI: TR, $3000.0 \mathrm{~ms}$; TE, $22 \mathrm{~ms}$, matrix, $128 \times 128$, depth of stratum, $1.0 \mathrm{~mm}$; interlamellar spacing, $0.00 \mathrm{~mm}$; FOV, $3.5 \times 3.5 \mathrm{~cm}$.

Regions of interest (ROIs) for the MRI parameters were subjectively selected based on abnormal MRI signals by two independent researchers (GY and LLF). To ensure accuracy, all ROIs were selected and measured based on histological findings. Rs-T2WI (relative square in the T2WI sequence), rs-SWI and rs-DWI were calculated as follows: The area of abnormal signals of traumatic region/the total area of the traumatized hemisphere $\mathrm{x} 100$. The rs-ADC was also calculated as: The ADC value of ROI/the ADC value of the mirror region $\mathrm{x} 100$.

Pathological observation. After euthanasia, the ventricles of rats were infused with $4 \%$ paraformaldehyde and the brains were harvested and fixed with $4 \%$ paraformaldehyde for $24 \mathrm{~h}$ at room temperature. Coronal sections of the damaged brain regions were prepared at 5- $\mu \mathrm{m}$ thick and embedded with paraffin. Haematoxylin and eosin (H\&E) staining was performed for each sample at room temperature, and the slices were observed using an optical microscope (magnification, $\mathrm{x} 4$ ) (DMi1; Leica Microsystems GmbH).

For coronal sections prepared for immunohistochemical staining, they were first rehydrated in a descending ethanol gradient and then treated with trypsin antigen retrieval buffer (Sigma-Aldrich; Merck KGaA) at $37^{\circ} \mathrm{C}$ for $20 \mathrm{~min}$. Then the sections were blocked with $10 \%$ bovine serum albumin (Hyclone; GE Healthcare Life Sciences) at room temperature for $20 \mathrm{~min}$, following which AQP4 expression was detected using mouse monoclonal antibodies at $4^{\circ} \mathrm{C}$ overnight (cat. no. ab128906; 1:200; Abcam) and a horseradish peroxidase-conjugated anti-mouse 
antibody at room temperature for $3 \mathrm{~h}$ (cat. no. KL-F0983R; 1:100; Shanghai Kanglang Biotechnology Co., Ltd.), followed by color development with diaminobenzidine tetrahydrochloride (Dako; Agilent Technologies, Inc.). The slices were subsequently counterstained at room temperature for $1 \mathrm{~min}$ with hematoxylin. The results of AQP4 expression were quantified in integrated optical density using the Image-Pro Plus 6.0 software (Media Cybernetics, Inc.).

For double labeling immunofluorescence staining, sections were prepared as above, and then incubated with anti-AQP4 (1:200; cat. no. 16473; Wuhan Sanying Biotechnology) and anti-glial fibrillary acidic protein antibodies (1:200; cat. no. 14214; Wuhan Sanying Biotechnology) at $4^{\circ} \mathrm{C}$ overnight and exposed to DyLight 488 (1:200; cat. no. ZB.2301; Wuhan Sanying Biotechnology) and Alexa ${ }^{\circledR}$ Fluor 555 labeled secondary antibodies at room temperature for $3 \mathrm{~h}(1: 100$; cat. no. ZB.1301; Wuhan Sanying Biotechnology). After counterstaining with DAPI $(0.1 \mu \mathrm{g} / \mathrm{ml})$ at room temperature for $15 \mathrm{~min}$, slices were observed using a fluorescence microscope (magnification, x600; DM-IRB; Leica Microsystems GmbH).

For electron microscopy, samples were embedded with Spurr resin at room temperature overnight and sections were treated using lead-uranium double staining. Sections $(50 \mathrm{~nm})$ were prepared using a ultramicrotome, following which they were fixed with $2 \%$ glutaraldehyde at room temperature for $24 \mathrm{~h}$ and then dehydrated with ethanol gradient. Slices were stained with $3 \%$ uranium acetate dye solution at room temperature for $30 \mathrm{~min}$, followed by $2 \%$ lead citrate for $30 \mathrm{~min}$ at room temperature. The ultrastructures of tissue sections were observed under an EX_2000 transmission electron microscope (JEOL, Ltd.).

Statistical analysis. All experiments were repeated at least 3 times. The results are expressed as the mean \pm standard deviation. Statistical analysis was performed using a one-way ANOVA followed by Duncan's post hoc test using SPSS software (version 24.0; IBM Corp.). $\mathrm{P}<0.05$ was considered to indicate a statistically significant result.

\section{Results}

AQP4 expression and distribution after brain trauma. The efficacy of siRNA was demonstrated using western blot analysis, and the results indicated that AQP4 expression was downregulated after transfection with siRNA (Fig. S1). Western blot analysis indicated that AQP4 protein expression was significantly increased in the trauma and placebo groups at 6 and $12 \mathrm{~h}$ after injury compared with their respective control groups (Fig. 1). AQP4-RNAi injection inhibited the increase of AQP4 expression at 6 and $12 \mathrm{~h}$ after injury, as AQP4 expression was significantly decreased in the RNAi group compared with the placebo group at 6 and $12 \mathrm{~h}$ (Fig. 1).

Immunohistochemical staining also indicated that AQP4 expression was upregulated after injury, with its expression levels being the highest at $12 \mathrm{~h}$ post injury. In the RNAi group, the AQP4 expression was significantly suppressed at 6 and $12 \mathrm{~h}$ compared with the trauma group, (Fig. 2). The double labeling immunofluorescence staining also indicated the location of the AQP4 protein (Fig. 3; white arrows). In the
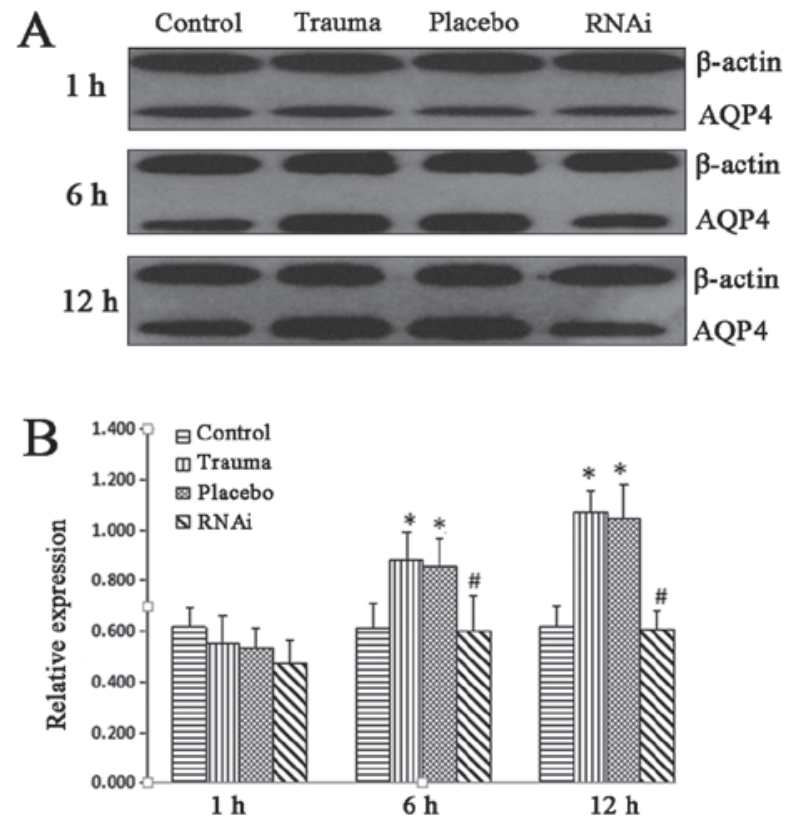

Figure 1. Western blot analysis of AQP-4 expression. Brain tissue was harvested and AQP-4 protein expression was measured using western blot analysis. (A) AQP-4 protein expression in the control, trauma, placebo and RNAi groups. (B) Western blot analysis of AQP-4 was quantified and data were expressed as the ratio of AQP-4 to $\beta$-actin. The data are presented as the mean \pm standard deviation from at least three independent experiments. ${ }^{*} \mathrm{P}<0.05$ vs. control group. ${ }^{\#} \mathrm{P}<0.05$ vs. trauma group. AQP-4, aquaporin-4; RNAi, RNA interference.

control group, a small amount of AQP4 protein was revealed around the gliocytes and endothelial cells. In the trauma group, AQP4 expression was indicated to be isolated around endothelial cells at $1 \mathrm{~h}$ after injury and around gliocytes foot processes $6 \mathrm{~h}$ after injury. A period of $12 \mathrm{~h}$ after injury, AQP4 was observed to be widely expressed around gliocytes and endothelial cells.

Early MRI changes after brain trauma. The MRI images indicated that there was no sign of edema in the control group (Fig. 4). However, in the trauma group, rs-DWI and rs-T2WI increased at $12 \mathrm{~h}$ post injury, with the expression ratio highest at $12 \mathrm{~h}$. R-ADC was increased and peaked at $6 \mathrm{~h}$, decreased and reached the lowest point at $12 \mathrm{~h}$ (Fig. 4). A similar trend was observed in the placebo group (Fig. 4). After treatment with AQP4-RNAi, compared with trauma and placebo groups, T2WI, rs-DWI and rs-SWI were observed to be significantly decreased from $12 \mathrm{~h}$, whilst r-ADC was indicated to be decreased at $6 \mathrm{~h}$ (Fig. 4).

Edema progression after brain trauma. In the placebo (data not shown) and trauma groups, edema was observed around the vascular endothelia, and mild cytotoxic edema was also indicated in gliocytes at $1 \mathrm{~h}$ after injury. Edema continued to increase over time in trauma groups (Fig. 5). In the trauma group, vasogenic and cytotoxic edema was observed at $6 \mathrm{~h}$ post injury and reached the highest level at $12 \mathrm{~h}$ post injury, and $H \& E$ staining revealed vasogenic and cytotoxic edema (Fig. 5A-a-c). In the RNAi group, the remission of edema was observed at $6 \mathrm{~h}$, and decreased further at $12 \mathrm{~h}$ after injury (Fig. 5A-e-f). 
A
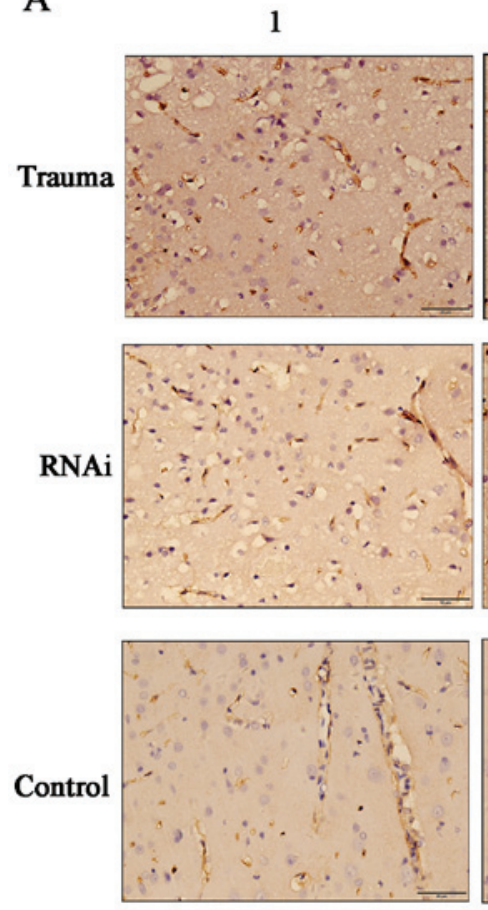

6
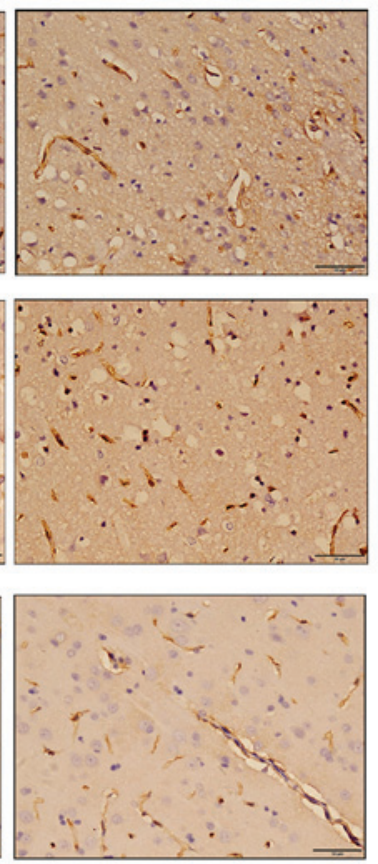

12
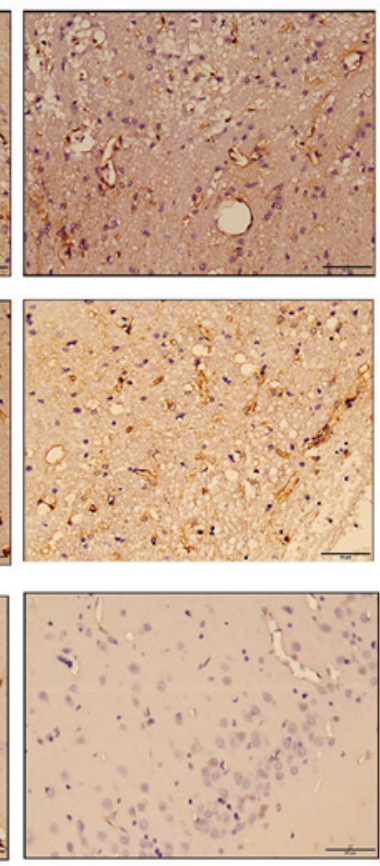

B

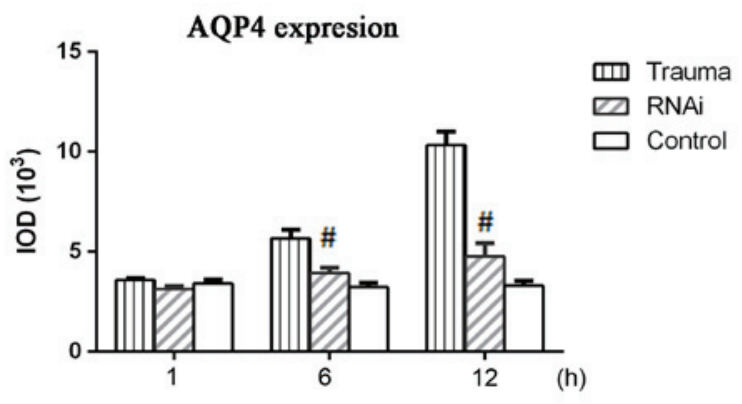

Figure 2. Immunohistochemical staining of AQP-4. Brain tissue was harvested and sections were prepared for immunohistochemical staining. (A) AQP-4 immunohistochemical staining (magnification, $\mathrm{x} 400$ ). (B) The results of immunohistochemistry staining were quantified in IOD. The data are presented as the mean \pm standard deviation from at least three independent experiments. ${ }^{\#} \mathrm{P}<0.05$ vs. trauma group. AQP-4, aquaporin-4; IOD, integrated optical density; RNAi, RNA interference.

TEM images indicated these changes in further detail. In the trauma group, discontinuity of endothelia (Fig. 5B-a; black arrow; blue star, blood vessels), swelling of mitochondria (yellow arrow) and endoplasmic reticulum (white arrow) were revealed at $1 \mathrm{~h}$ after injury. At $6 \mathrm{~h}$, chromatin aggregation was observed (Fig. 5B-b; white arrow), and edema was indicated in the mitochondria (black arrow) and around blood vessels (blue star). The vasogenic and cellular edema were identified at $12 \mathrm{~h}$ post injury (Fig. 5B-c; blue star). Endothelial breakage (black arrow) and the destruction at ridges of mitochondria (white arrow) were observed. Compared with the trauma group, the extent of edema in the RNAi group was markedly reduced from $6 \mathrm{~h}$ post injury. There was no obvious change in the RNAi group compared with the trauma group at $1 \mathrm{~h}$ post injury. However, at $6 \mathrm{~h}$ post injury, it was revealed that chromatin aggregation was decreased (Fig. 5B-e; white arrow) and the swelling of mitochondria (black arrow) and blood vessels (blue star) were all decreased. At $12 \mathrm{~h}$, this effect was increased in the RNAi group, as the discontinuity of endothelia

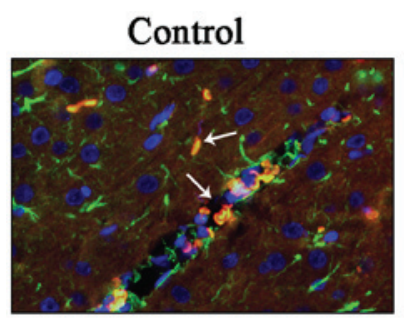

Trauma-6h

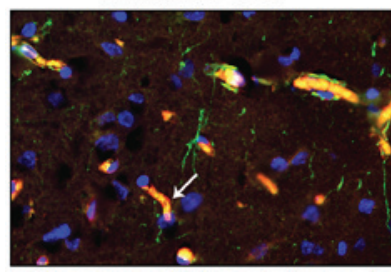

Figure 3. Double labeling immunofluorescence staining of AQP-4 (magnification, x600). Sections were incubated with anti-AQP-4 and anti-GFAP antibodies, and counterstained using DAPI. White arrow, positive staining of AQP-4; AQP-4, aquaporin-4; GFAP, glial fibrillary acidic protein. 

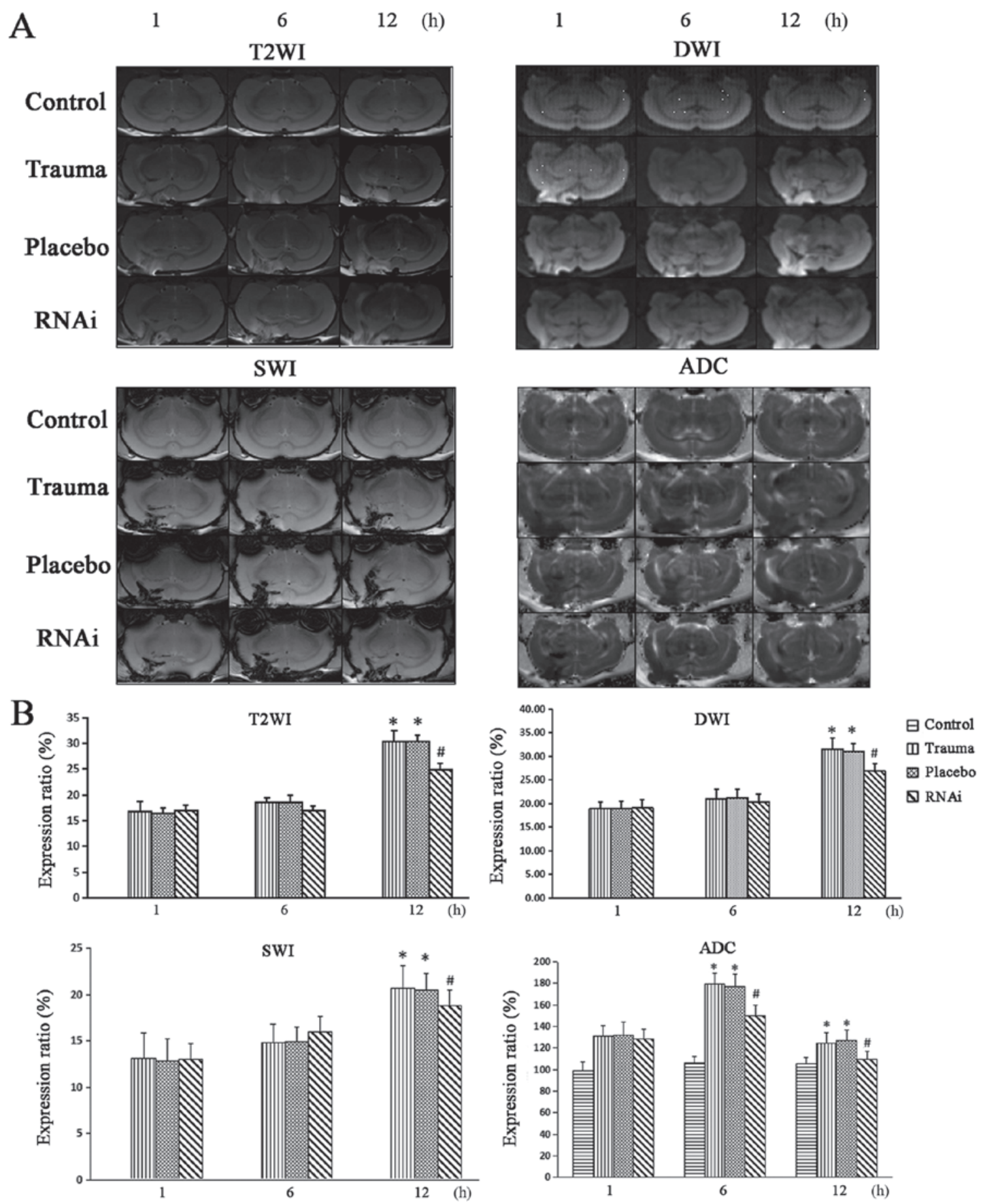

Figure 4. Multi-modal MRI images. All rat groups were imaged using a 7.0t MRI including DWI, T2WI, SWI and ADC sequences. (A) MRI images of rats brain. (B) Ratio of the area with abnormal signals in all sequences (the ratio of control group is 0 in the T2WI, DWI and SWI sequences). The data are presented as the mean \pm standard deviation from at least three independent experiments. ${ }^{*} \mathrm{P}<0.05$ vs. control group. ${ }^{*} \mathrm{P}<0.05$ vs. trauma group. DWI, diffusion-weighted MRI; T2WI, T2 weighted image; SWI, susceptibility weighted image; ADC, apparent diffusion coefficient; RNAi, RNA interference.

(Fig. 5B-f; black arrow) was alleviated as well as the swelling of mitochondria (black arrow) and blood vessels (blue star).

\section{Discussion}

The pathological changes that follow TBI are complex, and the early stage of brain trauma is a critical period for planning treatment $(6,14)$. A report has indicated that during the early stages of brain trauma, the primary site of brain trauma predominantly exhibits cytotoxic edema and the surrounding region is dominated by vasogenic edema (15). However, the features of brain edema following TBI are yet to be fully determined.

The pathological process of TBI contributes to MRI value changes and can also be monitored using MRI (16). However, the accurate determination of the development and pathological type (e.g. cytotoxic or vasogenic) of brain edema following TBI is difficult based on a single sequence of MRI assessments. Considering the differences between the treatments of cytotoxic and vasogenic brain edema, it is important to distinguish 


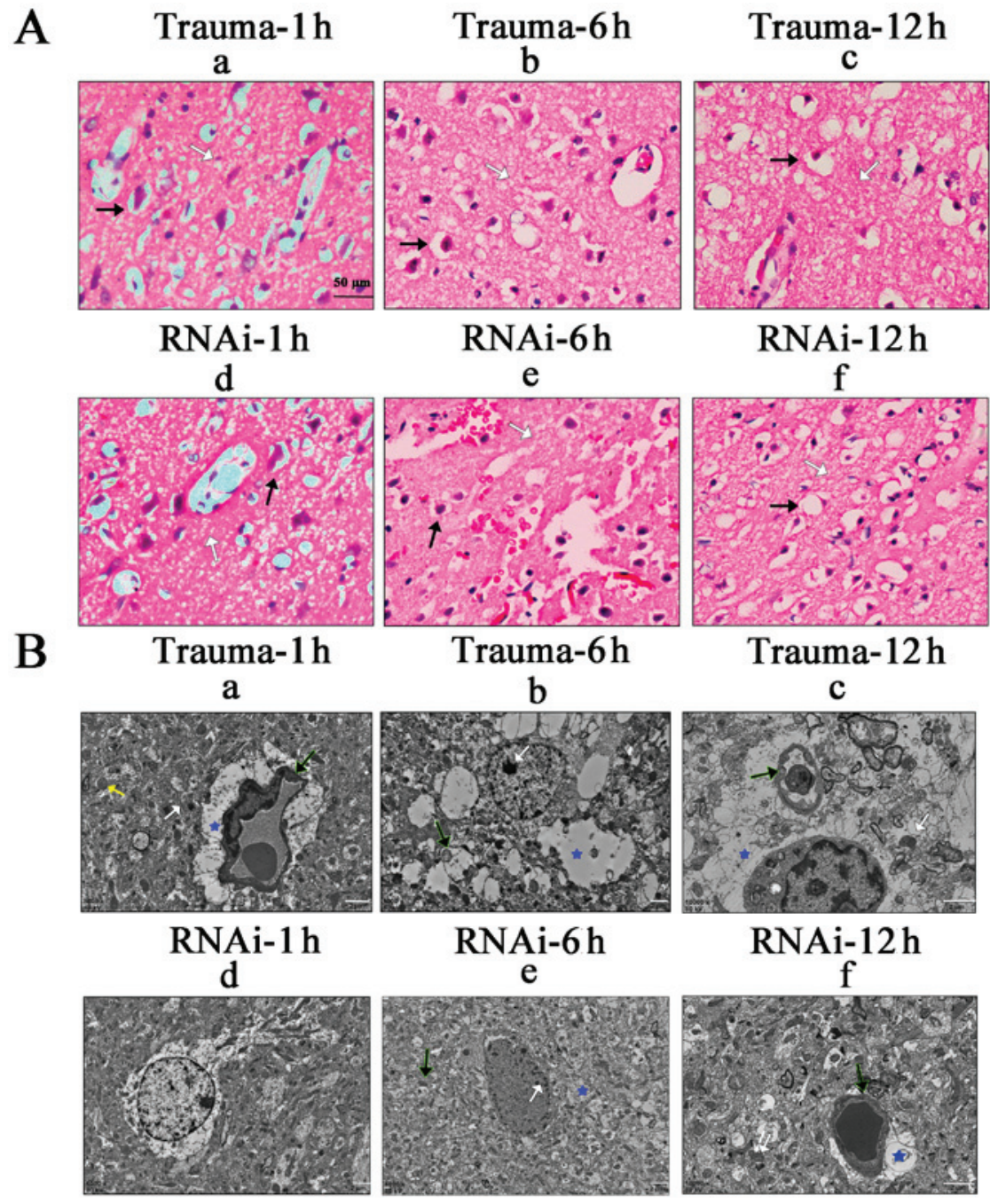

Figure 5. Pathological observation of brain tissue. (A) Haematoxylin and eosin staining of brain tissue. White arrow, vasogenic edema; black arrow, cytotoxic edema. Scale bar, $50 \mu \mathrm{m}$. (B) SEM images of brain tissue. Yellow arrow, endoplasmic reticulum; black arrow, endothelial; white arrow, mitochondria; blue star, vasogenic edema. Scale bar, $2 \mu \mathrm{m}$. SEM, scanning electron microscopy; RNAi, RNA interference.

the type of edema prior to the initiation of treatment (17). The multi-modal MRI allows more information to be gained, and also allows for an increased accuracy of diagnosis (7).

Compared with DWI alone, ADC can obtain a highly accurate diagnosis by reducing the T2 shine effect $(18,19)$. The high signal of DWI and ADC indicated vasogenic edema, while the high signal of DWI combined with a low signal of ADC indicated cytotoxic edema $(18,19)$. The SWI signal cannot distinguish edema from normal brain tissue. However, the damage area may be clearly visible (19). T2WI can indicate the extent and range of edema, however, it is only sensitive to vasogenic edema (7). According to the results of the present study, the multimodal MRI, which composited T2WI, DWI, $\mathrm{ADC}$ and DWI sequences, may be much more sensitive and useful in monitoring the extent and range of edema.

AQP4 has been previously reported to serve a role in the negative effects of brain edema (20). Many brain pathologies, including stroke, trauma and hemorrhage, have been associated with the dysfunction of AQP4 and resultant brain edema $(5,21)$. However, the function of AQP4 in maintaining water homoeostasis is complex. Tang et al (21) demonstrated that cytotoxic edema and astrocyte apoptosis was reduced due to the attenuation of downregulation of AQP4. Vindedal et al (22) reported that basal brain water content was increased in mice exhibiting a complete loss of AQP4 (global Aqp4-/- mice). Previous research has demonstrated that AQP4-overexpressing mice exhibit an accelerated progression of cytotoxic brain swelling after acute water intoxication, and it was concluded that AQP4 expression could limit the rate of brain water accumulation (23). In a previous study, RNAi technology was indicated to be effective in downregulating the expression of AQP4 in astrocytes, and consequently reducing ischemia-related brain edema (5). This treatment has also been revealed to exhibit a beneficial effect on brain edema after long term TBI (2). The expression of AQP4 has also been reported to be upregulated $24 \mathrm{~h}$ after TBI, however in this study, the edema was already severe (24). To further study the changes in AQP4 expression during edema and seek early intervention methods, AQP4 expression was measured in the current study at the early stage of TBI $(<12 \mathrm{~h})$.

Our previous research has indicated that the expression of AQP4 exhibited a complex pattern following TBI, with AQP4 decreasing then increasing (25). These results suggested that the downregulation of AQP4 was a result of vasogenic edema and the upregulation of AQP4 may induce cellular edema (2). 
Bias was present during the in vivo experiments as in some MRI images, the placebo group appeared to exhibit worse injury than the trauma group despite the statistical analysis indicating no significant difference between the two groups. To achieve stable transfection of siRNA in vivo and avoid the influence of technical bias, a relatively large sample size was used (120 rats) in the current study. Brain edema was indicated to be significantly reduced at 6 and $12 \mathrm{~h}$ and not at $1 \mathrm{~h}$ post TBI following treatment with RNAi. These results suggested that at the early stages of TBI $(1 \mathrm{~h})$, AQP4 was redistributed around the endothelial cells and caused vasogenic edema rather than upregulating protein expression. At the mid-early stage of TBI (6 to $12 \mathrm{~h}$ ), the protein expression of AQP4 was upregulated and redistributed on the surface of gliocytes, resulting in the exacerbation of cytotoxic edema. Therefore, the AQP4 RNAi treatment may downregulate the expression of AQP4 and reduce brain edema from 6 to $12 \mathrm{~h}$ post TBI.

The results of the present study demonstrated that the multimodal MRI was a useful and reliable tool in the evaluation of brain edema following TBI. The AQP4 RNAi treatment reduced brain edema at the early stages of TBI $(<12 \mathrm{~h})$. In conclusion, the current study indicated the features, mechanism and radiological evaluation methods of brain edema at early stages of TBI, and provides new evidence for the importance of early intervention during and following TBI.

\section{Acknowledgements}

Not applicable.

\section{Funding}

The current study was funded by the Finance science and technology project of Hainan Province (grant no. ZDYF2018175), the National Natural Science Foundation of China (grant no. 81160181), Key Projects of Chongqing Health and Family Planning Commission (grant no. 2016ZDXM040), Technology Program of Banan District Chongqing Science and Technology Commission (grant no. 2018-25) and Industry Project of Hainan Health Department (grant no. 16A200107).

\section{Availability of data and materials}

The datasets used and/or analyzed during the current study are available from the corresponding author on reasonable request.

\section{Authors' contributions}

YG, LL and HL conceived and designed the study. YG and LL performed the experiments. YG and JC analyzed the experimental data. YG, JC and HL wrote the manuscript. YG and HL reviewed and edited the manuscript. All authors read and approved the final manuscript.

\section{Ethics approval and consent to participate}

The current study was approved by the Ethics Committee of the First Affiliated Hospital of Hainan Medical University (approval no. 2016054; Hainan, China).

\section{Patient consent for publication}

Not applicable.

\section{Competing interests}

The authors declare that they have no competing interests.

\section{References}

1. Stein DM, Feather CB and Napolitano LM: Traumatic brain injury advances. Crit Care Clin 33: 1-13, 2017.

2. Fukuda AM, Adami A, Pop V, Bellone JA, Coats JS, Hartman RE, Ashwal S, Obenaus A and Badaut J: Posttraumatic reduction of edema with aquaporin-4 RNA interference improves acute and chronic functional recovery. J Cereb Blood Flow Metab 33: $1621-1632,2013$.

3. Marmarou A, Fatouros PP, Barzó P, Portella G, Yoshihara M, Tsuji O, Yamamoto T, Laine F, Signoretti S, Ward JD, et al: Contribution of edema and cerebral blood volume to traumatic brain swelling in head-injured patients. J Neurosurg 93: 183-193, 2000.

4. Kunkel A, Filik R, Mackenzie IG and Leuthold H: Task-dependent evaluative processing of moral and emotional content during comprehension: An ERP study. Cogn Affect Behav Neurosci 18: 389-409, 2018.

5. Nicchia GP, Frigeri A, Liuzzi GM and Svelto M: Inhibition of aquaporin- 4 expression in astrocytes by RNAi determines alteration in cell morphology, growth, and water transport and induces changes in ischemia-related genes. FASEB J 17: 1508-1510, 2003.

6. Lescot T, Fulla-Oller L, Po C, Chen XR, Puybasset L, Gillet B, Plotkine M, Meric P and Marchand-Leroux C: Temporal and regional changes after focal traumatic brain injury. J Neurotrauma 27: 85-94, 2010.

7. Maegele M, Stuermer EK, Hoeffgen A, Uhlenkueken U, Mautes A, Schaefer N, Lippert-Gruener M, Schaefer U and Hoehn M: Multimodal MR imaging of acute and subacute experimental traumatic brain injury: Time course and correlation with cerebral energy metabolites. Acta Radiol Short Rep 4: $2047981614555142,2015$.

8. Xu S, Zhuo J, Racz J, Shi D, Roys S, Fiskum G and Gullapalli R: Early microstructural and metabolic changes following controlled cortical impact injury in rat: A magnetic resonance imaging and spectroscopy study. J Neurotrauma 28: 2091-2102, 2011.

9. Tang G and Yang GY: Aquaporin-4: A potential therapeutic target for cerebral edema. Int J Mol Sci 17: pii: E1413, 2016.

10. Manley GT, Fujimura M, Ma T, Noshita N, Filiz F, Bollen AW, Chan P and Verkman AS: Aquaporin-4 deletion in mice reduces brain edema after acute water intoxication and ischemic stroke. Nat Med 6: 159-163, 2000.

11. Vajda Z, Promeneur D, Dóczi T, Sulyok E, Frøkiaer J, Ottersen OP and Nielsen S: Increased aquaporin-4 immunoreactivity in rat brain in response to systemic hyponatremia. Biochem Biophys Res Commun 270: 495-503, 2000.

12. McIntosh TK, Vink R, Noble L, Yamakami I, Fernyak S, Soares H and Faden AL: Traumatic brain injury in the rat: Characterization of a lateral fluid-percussion model. Neuroscience 28: 233-244, 1989.

13. Badaut J, Fukuda AM, Jullienne A and Petry KG: Aquaporin and brain diseases. Biochim Biophys Acta 1840: 1554-1565, 2014.

14. Wei XE,Zhang YZ, Li YH,Li MH and Li WB: Dynamics of rabbit brain edema in focal lesion and perilesion area after traumatic brain injury: A MRI study. J Neurotrauma 29: 2413-2420, 2012.

15. Tourdias T, Dragonu I, Fushimi Y, Deloire MS, Boiziau C, Brochet B, Moonen C, Petry KG and Dousset V: Aquaporin 4 correlates with apparent diffusion coefficient and hydrocephalus severity in the rat brain: A combined MRI-histological study. Neuroimage 47: 659-666, 2009.

16. Hudak AM, Peng L, Marquez de la Plata C, Thottakara J, Moore C, Harper C, McColl R, Babcock E and Diaz-Arrastia R: Cytotoxic and vasogenic cerebral oedema in traumatic brain injury: Assessment with FLAIR and DWI imaging. Brain Inj 28: 1602-1609, 2014.

17. Gatidis S, Schmidt H, Martirosian P, Nikolaou K and Schwenzer NF: Apparent diffusion coefficient-dependent voxelwise computed diffusion-weighted imaging: An approach for improving SNR and reducing T2 shine-through effects. J Magn Reson Imaging 43: 824-832, 2016. 
18. Oshio K, Okuda S and Shinmoto H: Removing ambiguity caused by T2 Shine-through using weighted diffusion subtraction (WDS). Magn Reson Med Sci 15: 146-148, 2016

19. Hsu CC, Watkins TW, Kwan GN and Haacke EM: Susceptibility-weighted imaging of Glioma: Update on current imaging status and future directions. J Neuroimaging 26: 383-390, 2016

20. Chen JQ, Zhang CC, Jiang SN, Lu H and Wang W: Effects of aquaporin 4 knockdown on brain edema of the uninjured side after traumatic brain injury in rats. Med Sci Monit 22: 4809-4819, 2016.

21. Tang G, Liu Y, Zhang Z, Lu Y, Wang Y, Huang J, Li Y, Chen X, Gu X, Wang Y, et al: Mesenchymal stem cells maintain blood-brain barrier integrity by inhibiting aquaporin-4 upregulation after cerebral ischemia. Stem Cells 32: 3150-3162, 2014.

22. Vindedal GF, Thoren AE, Jensen V, Klungland A, Zhang Y, Holtzman MJ, Ottersen OP and Nagelhus EA: Removal of aquaporin-4 from glial and ependymal membranes causes brain water accumulation. Mol Cell Neurosci 77: 47-52, 2016.
23. Yang B, Zador Z and Verkman AS: Glial cell aquaporin-4 overexpression in transgenic mice accelerates cytotoxic brain swelling. J Biol Chem 283: 15280-15286, 2008.

24. Higashida T, Kreipke CW, Rafols JA, Peng C, Schafer S, Schafer P, Ding JY, Dornbos D III, Li X, Guthikonda M, et al: The role of hypoxia-inducible factor-1 $\alpha$, aquaporin- 4 and matrix metalloproteinase-9 in blood-brain barrier disruption and brain edema after traumatic brain injury. J Neurosurg 114: 92-101, 2011

25. Lu H, Lei XY, Hu H and He ZP: Relationship between AQP4 expression and structural damage to the blood-brain barrier at early stages of traumatic brain injury in rats. Chin Med J (Engl) 126: 4316-4321, 2013.

(i)(9) This work is licensed under a Creative Commons Attribution-NonCommercial-NoDerivatives 4.0 International (CC BY-NC-ND 4.0) License. 